\title{
Efficient blind spectrum sensing for cognitive radio networks based on compressed sensing
}

\author{
Shancang Li ${ }^{1,2^{*}}$, Xinheng Wang ${ }^{2}$, Xu Zhou $^{3}$ and Jue Wang ${ }^{1}$
}

\begin{abstract}
Spectrum sensing is a key technique in cognitive radio networks (CRNs), which enables cognitive radio nodes to detect the unused spectrum holes for dynamic spectrum access. In practice, only a small part of spectrum is occupied by the primary users. Too high sampling rate can cause immense computational costs and sensing problem. Based on sparse representation of signals in the frequency domain, it is possible to exploit compressed sensing to transfer the sampling burden to the digital signal processor. In this article, an effective spectrum sensing approach is proposed for CRNs, which enables cognitive radio nodes to sense the blind spectrum at a sub-Nyquist rate. Perfect reconstruction from fewer samples is achieved by a blind signal reconstruction algorithm which exploits $\ell_{p}$-norm $(0<p<1)$ minimization instead of $\ell_{1}$ or $\ell_{1} / \ell_{2}$ mixed minimization that are commonly used in existing signal recovery schemes. Simulation results demonstrated that the $\ell_{p}$-norm spectrum reconstruction scheme can be used to break through the bandwidth barrier of existing sampling schemes in CRNs.
\end{abstract}

\section{Introduction}

In cognitive radio networks (CRNs), spectrum sensing aims to identify the frequency support of a signal, which consists of spectrum intervals that the power of the signal exceeds that of noise [1]. Recently, many researchers have focused their attentions on spectrum sensing in CRNs, in which the cognitive radio (CR) nodes are able to perform the wideband spectrum sensing to detect the unoccupied frequency bands for temporal using. As a very promising technology in CRNs [2,3], the compressed sensing theory can be used to alleviate the dynamic spectrum sensing problem by blindly detecting the spectrum holes [3-5].

The basic idea behind compressed sensing (CS in short) is to sample compressible signals at a lower rate than the traditional Nyquist, and then reconstruct these signals with compressed measurements [3]. In CS, the sampling and compression operations are combined into a low complexity compressed sampling [4], in which compressible signals can accurately be reconstructed from a set of random linear measurements by using nonlinear or convex reconstruction algorithms [6,7]. Typically,

\footnotetext{
*Correspondence: s.li@swansea.ac.uk

1 Key Laboratory of Biomedical Information Engineering of Ministry of Education, Xi'an Jiaotong University, Xi'an 710049, China

${ }^{2}$ College of Engineering, Swansea University, Swansea, SA2 8PP, UK

Full list of author information is available at the end of the article
}

the number of measurements in CS is much fewer than that in Nyquist sampling, thus leading to a significant reduction in sampling rates. Therefore, the requirements to analog-to-digital converter resource can be reduced significantly, which is of great importance for wideband communication systems [4]. Previously, a lot of CS-based techniques have been proposed [3,4]. The new CS theory is hoped to significantly reduce the sampling rate and computational costs at a CR node for compressible signals [8].

A compressible signal means that it can sparsely be represented in some basis, and can exactly be reconstructed only with a small set of random projections on an incoherent basis [8-10]. Recently, many research efforts have been done on random projection. The authors of [11] proposed a collaborative compressed spectrum sensing, where the compressed spectrum reconstruction is modeled with a gaussian process framework model. The authors of [12] investigate the problem of dynamic resource allocation in CRNs, where several CS-based techniques are used to detect occupied spectral bands from compressed measurements. For current CRNs, the CS has been used to alleviate the sampling bottleneck, which aims at decreasing the sampling rates for the acquisition of compressible signals $[13,14]$.

\section{Springer}


The CS techniques that have been used in spectrum sensing can be classified into two categories: (1) Convex relaxing-based methods, such as basis pursuit (BP) [15,16] and Dantzig Selector [17]; (2) Greedy algorithmbased methods, such as matched pursuit (MP) and its variants [18]. Recently, many improved MP-based methods have been reported such as orthogonal matched pursuit [19], regularized orthogonal matched pursuit [20], compressive sampling matching pursuit [21], and so forth. Actually, the former or its variants can get higher reconstruction accuracy, however it may cause expensive computation costs. The greedy algorithm-based methods have less computing complex, however the reconstruction accuracy is limited compared with the convex programming. The basis pursuit denoising is commonly used in signal processing due to its additional denoising performance advantage $[16,22]$. The advantages of the greedy algorithm-based approaches are fast, stable, uniform guarantees, however it requires a slightly stronger condition on the restricted isometry property (RIP) condition than first category [23].

The spectrum sensing in CRNs faces three main technical challenges: (1) The sampling rate, too high sampling rate may cause very high cost of signal processing and storage; (2) The design of radio front-end is very difficult, the computation intensive energy or feature detection operations are applied in many existing spectrum sensing methods. However, by using CS-based approach, the spectrum detection can be simplified; and (3) highspeed DSP that operates at or above the Nyquist rate is used in conventional spectrum estimation, which may cause failure of exactly signals reconstruction because of the high requirement on spectrum sensing timing windows [3].

In this article, we aim at developing an effective CSbased spectrum sensing approach at affordable complexity. First, we take a multi-coset scheme to decompose the spectrum in CRNs. One of the goals of each CR is to effectively detect the unused spectrum holes while the spectrum sparsity is known a priori for the dynamic spectrum access of CRs. The cognitive spectrum sensing is decomposed into two stages: spectrum sensing and spectrum reconstruction. In spectrum sensing, the sensing time to find the spectrum holes is critical for the 'cognition' of the CRs. On the other hand, the spectrum recovery requires better anti-noise performance. In order to cope with these challenges, we focus our works on the following issues:

1. A CS-based spectrum sensing scheme is proposed which can adaptively sense the blind occupied bands with a sampling rate lower than that of Nyquist;

2. In the spectrum reconstruction, we proposed an improved block sparse signal model, in which an approximate $\ell_{p}$-norm $(0<p<1)$ minimization is used to improve the reconstruction quality and speed spectrum.

3. To further enhance the performance and reconstruction speed, an iterative weighted scheme is proposed to approximate the $\ell_{p}$-norm optimization problem, by doing this the convergence speed can be enhanced in reconstruction.

Notation: For vectors/matrices the superscript, T, denotes transpose. $\mathbf{A}_{l, k}$ represents the $(l, k)$ th element of a matrix $\mathbf{A}$. $\|\mathbf{x}\|$ denotes the $\ell_{2}$-norm of vector $\mathbf{x}$. In general, $\|\mathbf{x}\|_{p}$ denotes the $\ell_{p}$-norm of $\mathbf{x}$ that is defined as $\|\mathbf{x}\|_{p}=$ $\left(\sum_{i=1}^{N}\left|\mathbf{x}_{i}\right|^{p}\right)^{1 / p}$. The common notations that summarized in Table 1 is used in this article.

\section{System models \\ Signal model}

In practice, a signal can always be sparsely or near-sparsely represented on a transform domain [24]. An $N$-length signal $\mathbf{x}$ can easily be described as

$$
\mathbf{x}=\Psi \theta
$$

where the $N \times 1$ vector $\theta$ is the $K$-sparse representation of $\mathbf{x}$, and $K$ is the number of nonzero elements of $\theta(K \ll N)$. $\Psi=\left\{\psi_{1}, \psi_{2}, \ldots, \psi_{N}\right\}$ is an $N \times N$ transform matrix, and $\psi_{i}(i=1 \ldots N)$ is the similarly sampled basis function.

\section{Table 1 Notation}

\begin{tabular}{ll}
\hline$x(t)$ & A continuous-time signal with finite energy \\
$x(f)$ & The Fourier transform of $x(t)$ \\
$x[n]$ & Bounded energy sequence \\
$\mathbf{v}$ & Vector \\
$\mathbf{v}_{i,}$ or $\mathbf{v}(i)$ & ith entry of $\mathbf{v}$ \\
$\mathbf{v}(f)$ & Vector that depends on a continuous parameter $f$ \\
$\boldsymbol{\Phi}$ & Matrix \\
$\boldsymbol{\Phi}_{i k}$ & $i k$ th entry of $\Phi$ \\
$\boldsymbol{\Phi}_{i}$ & $i$ th column of $\Phi$ \\
$\mathbf{r}_{t}$ & Samples vector of a signal at time domain \\
$\mathbf{r}_{f}$ & Samples vector of a signal at frequency domain \\
$\mathcal{F}$ & Non-zero frequency-domain support \\
$q_{1} L$ & a number of blocks and $L$ denotes the number of all blocks \\
$B, B_{i}$ & Bandwidth of sub-band, $B_{i}$ denotes the $i$-th sub-band \\
$Q$ & Threshold for blind spectrum reconstruction \\
$\|\mathbf{x}\|_{0}$ & $\ell_{0}$-norm of $\mathbf{x}$ \\
$\|\mathbf{x}\|_{p}$ & $\ell_{p}$-norm of $\mathbf{x}$, which is defined as $\|\mathbf{x}\|_{p}=\left(\sum_{i=1}^{N}\left|\mathbf{x}_{i}\right|^{p}\right)^{1 / p}$ \\
$(\mathbf{A})$ & Support of $\mathbf{A}$ \\
\hline $\mathcal{M}$ &
\end{tabular}


For a time window as $t \in\left[0, T_{0}\right], N$ samples are necessary for Nyquist theorem to exactly reconstruct the power spectrum density.

$$
\mathbf{x}=F \mathbf{r}_{t}
$$

where $\mathbf{F}$ is the discrete-time Fourier transform (DFT) matrix and $\mathbf{r}_{t}$ is the sample vector of a real-value signal $r(t)$ which needs to be reconstructed from samples $\mathbf{x}$.

As mentioned above, the $C S$ is able to accurately reconstruct signals only with a small portion of samples with size of $M(M \ll N)$

$$
\mathbf{y}=\Phi x
$$

in which y denotes an $M$-length measurement vector, and $\boldsymbol{\Phi}$ is the measurement matrix. The spectrum of $r(t)$ can accurately be reconstructed when the measurement y is available. Because $M \ll N$ so the sample rate can be reduced significantly. Here, we aim at developing a spectrum sensing scheme with fewer non-adaptive measurements where $\boldsymbol{\Phi}$ is well presented.

Each CR node is able to classify and estimate spectrum of a signal $r(t)$ by using the sample set $\mathbf{x}$ [3]. Suppose that the total frequency range is available as $B \mathrm{~Hz}$, then each $\mathrm{CR}$ node periodically senses the spectrum environment to find spectrum holes for opportunistic use [25]. Following assumptions are made:

1. The frequency boundaries are known to the CR and the bandwidth of the spectrum bands occupied by each CR is much less than $B$.

2. The number of bands $Q$ is known and their location are unknown to the $C R$ nodes. In a time burst, the locations and the number of bands $Q$ keep unchanged but may vary for different time bursts.

3. The signal power spectrum density (PSD) over each spectrum subband $B_{n}$ is smooth, however the PSD over two neighboring bands are independent.

\section{Signal sampling model and problem formulation}

For a time-continuous band-limited signal $r(t)$, its Fourier transform can be calculated as $X(f)=\mathbf{x}=\Psi \theta$, which is piecewise continuous in frequency $f$. We assume that the $r(t)$ is a band-limited signal in the non-zero frequencydomain support $\mathcal{F}$, and $X(f)=\mathbf{0}$ for $f \notin \mathcal{F}$.

We aim to exactly reconstruct $r(t)$ from a set of samples based on following constraints: (1) $r(t)$ is blind which means the locations of bands of $r(t)$ are not available in signal acquisition and reconstruction; (2) The sampling rate should be minimal for exactly reconstruction; (3) The signal can be reconstructed with a high probability even when ambient additive and white noise involved.

In Nyquist sampling, the sample sequence $x(n T)$ contains all the information about $r(t)$. However, in CS-based sampling the uniform grid is divided into $L$ consecutive sample blocks, which can implemented be by a constant set $C$. Assume the length of $C$ is $q$ and $C$ includes the indices of $q$ samples in each block ( e.g., $q_{i}$ denote the $i$ th element). Then, the sequence of samples can be represented as

$$
x_{c_{i}}[n]=\left\{\begin{array}{lr}
x(n T), & n=m L+c_{i}, m \in Z \\
0, & \text { otherwise }
\end{array}\right.
$$

For a set $C$ we have $0 \leq c_{1}<c_{2}<\cdots<c_{q} \leq L-1$, it is easy to understand that the sampling rate is $q / L \cdot r_{\mathrm{NYQ}}$ ( $r_{N Y Q}$ is the Nyquist sampling rate). Clearly, due to $q<L$ the sampling rate is less than $r_{\mathrm{NYQ}}$.

Having the estimation of frequency vector $\mathbf{r}_{f}$, it is necessary to detect the number and location of occupied bands of signal $r(t)$. We use a modulated wideband converter (MWC) [5], which aims at sampling wideband sparse signal at a rate lower than that of Nyquist. Figure 1 shows the MWC scheme.

Actually, the DFT $X_{c_{i}}(f)$ of $x_{c_{i}}[n]$ can be obtained according to Equation (5)

$$
\begin{aligned}
X_{c_{i}}(f) & =\sum_{n=-\infty}^{+\infty} x_{c_{i}}[n] e^{-j 2 \pi f n T} \\
& =\frac{1}{L T} \sum_{r=0}^{L-1} e^{\frac{j c_{i} r 2 \pi}{L}} X\left(f+\frac{r}{L T}\right)
\end{aligned}
$$

here $1 \leq i \leq q$ and $f \in \mathcal{F}$. Let $\mathbf{x}(f)$ denote vector $X_{c_{i}}(f),\left(c_{i} \in C\right)$ in frequency domain, then Equation (5) can be rewritten as

$$
\mathbf{y}(f)=\mathbf{A} \mathbf{x}(f)
$$

in which $\mathbf{y}(f)$ denotes a vector with length of $q$. It should be noted that the $i$ th element of $\mathbf{y}(f)$ is $X_{c_{i}}(f)$, and the matrix $\mathbf{A}$ is defined by

$$
\mathbf{A}_{i k}=\frac{1}{L T} e^{\frac{j c_{i} k 2 \pi}{L}}
$$

where the vector $\mathbf{x}(f)$ contains $L$ unknown components for each $f \in \mathcal{F}$

$$
\mathbf{x}_{i}(f)=X\left(f+\frac{i}{L T}\right), 0 \leq i \leq L-1, f \in \mathcal{F} .
$$

in which the the multi-coset sampling pattern $C$ includes all sampling time offsets, which are distinct and positive values less than $L$ [1]. It is crucial to properly select $L, q$, and $C$ such that $\mathbf{x}(f)$ can be reconstructed from Equation (6). In this case, the multi-coset sampling can provide the average sampling rate as $\frac{q}{L T}$ and the spectrum space can be extracted to bands with bandwidth $\frac{1}{L T}$.

Sequence $\mathbf{x}(f)$ is sparse since its Fourier property over frequency domain. However, under many scenarios the support set $I(\mathbf{x}(f))$ is not available. Fortunately, it is possible to find a unique sparsest solution, and the authors of [7] have proved that if $\mathbf{x}_{s}(f)$ is a solution for $\mathbf{y}(f)=\mathbf{A} \mathbf{x}(f)$ 


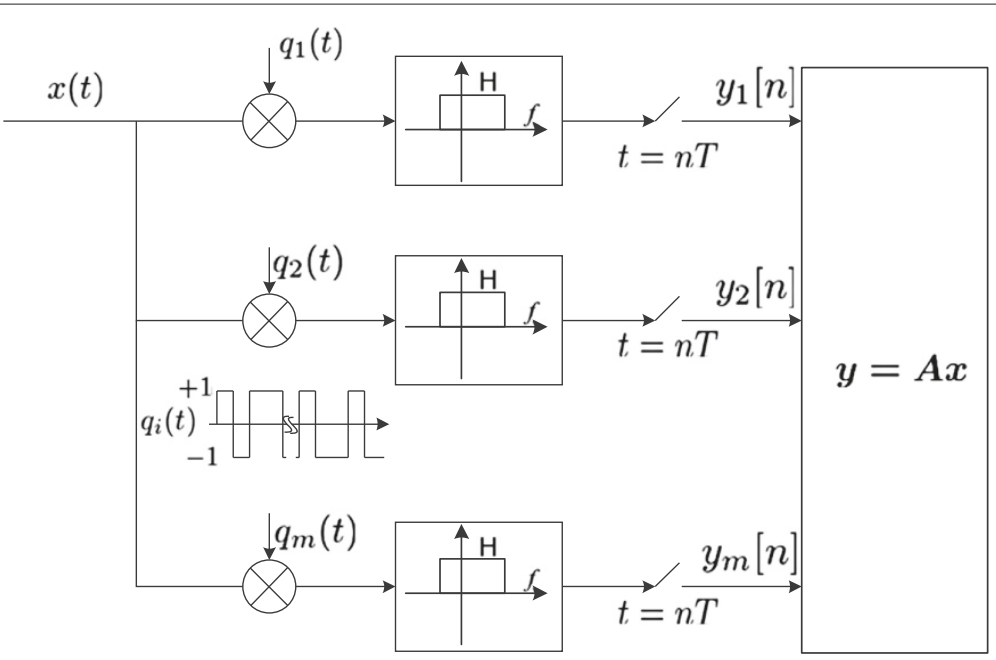

Figure 1 Compressed sampling scheme.

when $\left\|\mathbf{x}_{s}(f)\right\|_{0} \leq \sigma(\mathbf{A}) / 2$, then $\mathbf{x}_{s}(f)$ is the unique solution. Here, $\sigma(A)$ is the column rank of $A$. It is evident that the signal can perfectly be reconstructed when $\mathbf{x}(f)$ is $\sigma(A) / 2$-sparse.

Actually, the sparse level of $\mathbf{A}$ is related to the sampling coset pattern [1]. Because in multi-coset strategy the value of $Q, B$, and $T$ are available, thus the signals can be reconstructed with a high probability as a CS problem. For a signal $x(t) \in \mathcal{M}$, if $L \geq 1 / B T, C$ is a universal pattern, and $q \geq 2 Q$, then for every $f \in \mathcal{F}$, it is clear that vector $\mathbf{x}(f)$ is the unique solution of Equation (6).

\section{Compressed spectrum sensing}

For a normal signal, it is not difficult to find a sparse representation in a certain space [26]. Actually, signals involved in CRNs have been proved sparse in the frequency domain [26]. So, it is possible to find the unoccupied spectrum in CRNs with compressed spectrum sensing with a rate lower than Nyquist.

Let $\mathbf{r}$ denote the frequency response vector of signal $r(t)$ that can be obtained by $\mathbf{y}=\Phi F^{-1} \mathbf{r}$, in which $F$ is the Fourier transform matrix and $\Phi$ is the measurement matrix.

$$
\mathbf{r}=\underset{\mathbf{r}}{\arg \min }\|\mathbf{r}\|_{0} \text { s.t. }\left(\Phi F^{-1}\right) \mathbf{r}=\mathbf{y}
$$

It can be seen that Equation (9) is a non-convex problem. According to the RIP constraint we have $M \geq c \cdot M$. $\log (N / K)(c \in(0,1)$ is a universal constant). Equation (9) has a unique solution when Equation (10) holds

$$
\mathbf{r}=\underset{\mathbf{r}}{\arg \min }\|\mathbf{r}\|_{1} \text { s.t. }\left(\Phi F^{-1}\right) \mathbf{r}=\mathbf{y}
$$

Actually, Equation (10) is a second-order cone program and many software packages are available to solve this problem [9]. On the other hand, some variants of LASSO algorithm have been developed to deal with the noisy signals by minimizing the usual sum of squared errors.

$$
\mathbf{r}_{\mathrm{LASSO}}=\underset{\mathbf{r}}{\arg \min }\|\mathbf{r}\|_{1} \text { s.t. }\left\|\left(\Phi F^{-1}\right) \mathbf{r}-\mathbf{y}\right\|_{2} \leq \epsilon
$$

where $\epsilon$ bounds the noise in signals. A number of convex optimization software packages have been developed to solve the LASSO problem, such as cvx, SeDumi, Yalmip, and so on [9]. Recently, the authors of [9] improved Equation (11) with a weighted scheme of LASSO

$$
\mathbf{r}_{\mathrm{W}-\mathrm{LASSO}}=\underset{\mathbf{r}}{\arg \min }\|\mathbf{w r}\|_{1} \text { s.t. }\left\|\left(\Phi F^{-1}\right) \mathbf{r}-\mathbf{y}\right\|_{2} \leq \epsilon_{1}
$$

where signal $\mathbf{r}$ is separated into $K$ sub-vectors, and the weight vector $\mathbf{w}=\left[w_{1}, w_{2}, \ldots, w_{K}\right]^{T}$ can be calculated according to $p_{i}$-the subband power of the primary user existing in the $i$ th subband as $w_{i}=\frac{1}{p_{i}+\delta}$.

Previous methods take the sparsity of signals into consideration, and model the signal with CS theory in frequency domain based on $\ell_{1}$ or $\ell_{1} / \ell_{2}$-mixed minimization.

\section{Blind compressed spectrum sensing}

In CRNs, it is a very challenging topic to design a spectrum blind sampling-reconstruction system without knowing the locations of the bands. Actually, the bands occupied by different users may be discrete in CRNs, which makes it possible to design a spectrum-blind reconstruction scheme by using CS based on a preceding multi-coset model [27].

Let $\mathbf{r}_{t}$ denote the Nyquist sample sequence of $r(t)$, then we have $\mathbf{y}=\boldsymbol{\Phi} r_{t}$. It is possible to reconstruct the frequency response vector $\mathbf{r}_{f}$ of $r(t)$ from compressed samples $\mathbf{y}$. On the other hand, we have $\mathbf{r}_{f}=\boldsymbol{\Psi} \mathbf{r}_{t}$, in which $\boldsymbol{\Psi}$ 
is the DFT matrix. It is easy to understand that Equation (13) holds

$$
\mathbf{y}=\Phi \Psi^{-1} \mathbf{r}_{f}
$$

As discussed in Section "System models", the frequency response is sparse in CRNs, so Equation (13) can be solved with a two-step scheme: (1) use compressed measurements $\mathbf{y}$ to estimate the sparse frequency response $\mathbf{r}_{f}$ (actually this is an ill-posed problem); (2) reconstruct signal $r(t)$ according to the frequency response, which can be done by an inverse Fourier transfer.

It is easy to know that $\mathcal{F} \ll B$ which means $\mathbf{r}_{f}$ is sparse in the frequency domain. In order to solve the first problem, it can be formatted by CS theory

$$
\mathbf{r}_{f}=\underset{\mathbf{r}_{f}}{\arg \min }\left\|\mathbf{r}_{f}\right\|_{0} \text { s.t. }\left\|\left(\boldsymbol{\Phi} \Psi^{-1}\right) \mathbf{r}_{f}-\mathbf{y}\right\|_{2} \leq \epsilon_{1}
$$

In Section "Compressed spectrum sensing", we have summarized several methods that proposed to solve this problem. For simplicity we use $\mathbf{y}$ and $\mathbf{x}$ to denote $\mathbf{y}(f)$ and $\mathbf{x}(f)$, respectively. Then rewrite Equation (6) as

$$
\mathbf{y}=\mathbf{A} \mathbf{x}
$$

in which $\mathrm{A}=\mathbf{F}^{-1} \boldsymbol{\Phi} \boldsymbol{\Psi}$, and $\mathbf{F}$ denotes the DFT matrix of compressed sample vector $\mathbf{y}$.

Since $\mathbf{x}$ contains $M$ unknown elements for each $f$, and

$$
\mathbf{x}_{i}=X\left(f+\frac{i}{M T}\right), 0 \leq i \leq M-1, \forall f \in \mathcal{F}_{0}
$$

where $X(\cdot)$ is the Fourier transform of time shifted $r(t)$. As analyzed above, in a CRN every band contributes only a few non-zero value, so $\mathbf{x}$ is a sparse vector which makes it possible to use CS theory to reconstruct spectrum of signals.

Let $\sigma(A)$ denote the Kruskal-rank of $A$ [5]. When $\mathbf{x}$ is $\frac{\sigma(\mathbf{A})}{2}$-sparse then Equation (15) has a unique sparsest solution and the proof is given in [5]. It can be seen that reconstruction with high probability is possible for signal $\mathbf{x}$ that satisfies

$$
\mathbf{x} \text { is } \frac{\sigma(\mathbf{A})}{2}-\text { sparse, } \forall f \in \mathcal{F}_{0}
$$

As shown in Figure 1, if the number of sampling cosets $q$ is given, it can be proven that every signal $r(t) \in \mathcal{M}$ can perfectly be reconstructed by properly selecting parameters $Q, B$, and $T$.

Actually, if $M \leq 1 / B T$ and $q$ are greater than $Q$, then for every $f \in \mathcal{F}_{0}$ a unique $N$-sparse solution $\mathbf{x}$ is available according to Equation (15). According to Equation (16), it can be seen that $\mathbf{x}$ takes $M$ values of $X(f)$ by intervals of $1 / M T$, therefore the non-zero components are fewer than the number of bands in $\mathbf{x}(f)$. So we can say that $\mathbf{x}$ is $Q$ sparse. Kruskal-rank $\sigma(A)=q$, which implies that when $q \geq 2 Q B, \mathbf{x}$ can perfectly be reconstructed without know- ing any information about the locations of bands when $f \in \mathcal{F}_{0}$.

The blind reconstruction problem can be summarized as

$$
\begin{array}{r}
\mathbf{r}_{f}=\underset{\mathbf{r}_{f}}{\arg \min }\left\|\mathbf{r}_{f}\right\|_{1} \\
\text { s.t. } \quad\left\|\left(\boldsymbol{\Phi} \Psi^{-1}\right) \mathbf{r}_{f}-\mathbf{y}\right\|_{2} \leq \epsilon_{2}, \\
q \geq 2 Q B
\end{array}
$$

A number of CS reconstruction algorithms are available to solve this problem, and many $\ell_{1}$-norm and $\ell_{1} / \ell_{2}$-norm minimization-based approaches have been proposed for the reconstruction of sparse signal. However, according to the original idea of compressive sensing, $\ell_{p}$-norm minimization with $p<1$ can improve the recovery performance for signals that are less sparse [24,28,29]. On the other hand, the $\ell_{p}$-norm minimization offers good performance with reduced complexity.

\section{Weighted blind spectrum reconstruction (WBSR) Smoothly approximation of $\ell_{0}$-norm problem}

In CS theory, $\ell_{0}$-norm is the ideal minimization; however, it is an NP-hard problem to find out the sparest solution. In general, $\ell_{1}$-norm minimization, the contour of $\|\mathbf{x}\|_{1}=$ $k$, grows and touches the hyperplane $\boldsymbol{\Phi} \mathbf{x}=\mathbf{y}$, yielding a sparse solution as shown in Figure 2a. Unfortunately, this may cause a special situation that when the hyperplane is parallel to the contour of $\|\mathbf{x}\|_{1}=k$, infinite solutions can be derived, which may cause the unreliability of the system, as shown in Figure $2 \mathrm{~b}$.

On the other hand, the $\ell_{2}$-norm minimization may fail to work in CS. It is due to the fact that the contour of $\ell_{2}$ norm grows and touches the hyperplane, yielding an unsparse solution, which is no sense in solving our problem.

Our goal is to find out the sparest solution, which can be measured by using its $\ell_{0}$-norm pseudonorm. Unfortunately, the $\ell_{0}$-norm minimization problem is non-convex with combinatorial complexity. An effective signal reconstruction strategy is to solve the $\ell_{p}$-norm minimization problem as

$$
\begin{array}{r}
\text { minimize }\|\mathbf{x}\|_{p} \\
\text { s.t. } \boldsymbol{\Phi} \mathbf{x}=\mathbf{y}
\end{array}
$$

in which $0<p<1$. The $\ell_{p}$-norm minimization problem is non-convex. However, in $\ell_{p}$-norm minimization when the contour of $\|\mathbf{x}\|_{p}$ grows and touches the hyperplane $\boldsymbol{\Phi} \mathbf{x}=\mathbf{y}$ yields sparse solution, as shown in Figure 3c. The possibility that the contour will touch the hyperplane at another point is eliminated. 


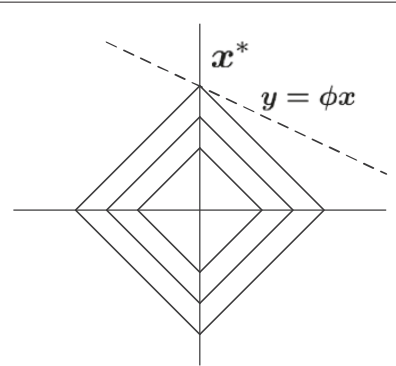

(a) $l_{1}$-norm

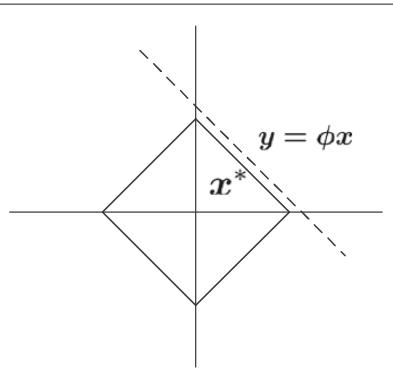

(b) ill $l_{1}$-norm

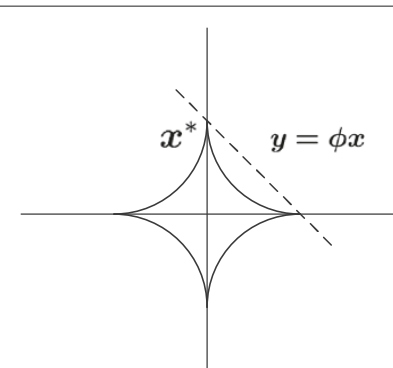

(c) $l_{p}$-norm $(0<p<1)$

Figure 2 Contours for $\ell_{1}$ and $\ell_{p}(0<\mathrm{p}<1)$ norm minimization.

In order to improve the convergence speed of the $\|\mathbf{x}\|_{p}$ problem, we build a differentiable and continuous function to approximate the $\|\mathbf{x}\|_{0}$ problem as

$$
\text { minimize }\|\mathbf{x}\|_{p}=\sum_{i=1}^{N}\left(1-e^{-x_{i}^{2} / 2 \sigma^{2}}\right)
$$

in which $\sigma$ is a very small constant that is used to guarantee the differentiability of Equation (20). It is easy to see that when $x_{i}$ is $0,1-e^{-x_{i}^{2} / 2 \sigma^{2}}$ approximates to zero. When $x_{i}$ is a non-zero value, then $1-e^{-x_{i}^{2} / 2 \sigma^{2}}$ approximates to 1 , so this function can approximate to $\ell_{0}$-norm problem smoothly.

In practice, the condition $M \geq c \cdot K \cdot \log (N / K)$ is restrictive for signal spectrum reconstruction. Based on the analysis in above sections, we reformulate the blind reconstruction problem with $\ell_{p}$-norm $(0<p<1)$ minimization approach as

$$
\begin{array}{r}
\underset{\mathbf{r}_{f}}{\operatorname{minimize}}\left\|\mathbf{r}_{f}\right\|_{p} \\
\text { s.t. } \quad\left\|\left(\boldsymbol{\Phi} \Psi^{-1}\right) \mathbf{r}_{f}-\mathbf{y}\right\|_{2} \leq \epsilon_{3}, \\
q \geq 2 Q B
\end{array}
$$

where $\left\|\mathbf{r}_{f}\right\|_{p}$ is with $0 \leq p<1$. For $p<1$, Equation (21) becomes a non-convex problem which has multiple solutions. If this problem can be solved with sufficient, then improved results can be available. We rewrite a smooth $\ell_{p}$-norm of $\mathbf{r}_{f}$ in Equation (22)

$$
\begin{array}{r}
\underset{\mathbf{r}_{f}}{\operatorname{minimize}} F\left(\mathbf{r}_{f}\right)=\sum_{i=1}^{N}\left(1-e^{-\left(\mathbf{r}_{f}\right)_{i}^{2} / 2 \sigma^{2}}\right) \\
\text { s.t. }\left\|\left(\boldsymbol{\Phi} \boldsymbol{\Psi}^{-1}\right) \mathbf{r}_{f}-\mathbf{y}\right\|_{2} \leq \epsilon_{4} \\
q \geq 2 Q B
\end{array}
$$

in which $\sigma>0$ is a small constant. Equation (22) can be solved with steepest-descent approach. It can be seen that this optimization problem can offer accurate spectrum reconstruction performance with reduced computation complexity.

\section{Signal reconstruction by $\ell_{p}$-norm minimization}

In this section, we will present an effective method to reconstruct signals by using $\ell_{p}$ minimization. From the CS theory, the solutions of $\mathbf{y}=\mathbf{A} \mathbf{r}_{f}$ can be represented as [29]

$$
\mathbf{r}_{f}=\mathbf{r}_{f}^{*}+\mathbf{V}_{r} \xi
$$

where $\mathbf{r}_{f}^{*}$ is a special solution to $A \mathbf{r}_{f}=\mathbf{y}$, which can be calculated as $\mathbf{r}_{f}^{*}=\mathbf{A}^{T}\left(\mathbf{A A}^{T}\right)^{-1} . \quad V_{r}$ is an $N \times(N-M)$ matrix and $\xi$ is a $N-M \times 1$ vector. $\mathbf{V}_{r}$ can be calculated by $Q R$ decomposition of matrix $A$, then we can further rewritten the $\ell_{p}$-norm problem as

$$
\underset{\mathbf{r}_{f}}{\operatorname{minimize}} F(\boldsymbol{\xi})=\sum_{i=1}^{N} 1-e^{-\left[\mathbf{r}_{f}^{*}(i)+\mathbf{v}_{i}^{T} \boldsymbol{\xi}\right]^{2} / 2 \sigma^{2}}
$$

where $\mathbf{v}_{i}^{T}$ is the $i$ th row of matrix $\mathbf{V}_{r}$. It is clear that Equation (24) is a differentiable function, so its gradient can be obtained [29], which reduce the problem size from $N$ to $N-M$. So, a number of existing approaches are available to solve Equation (24) as an unconstrained optimization problem, such as quasi-Newton [24], Broyden-Fletcher-Goldfarb-Shanno (BFGS) [29] and so on.

\section{Weighted approximation of $\ell_{p}$-norm algorithm}

Similar to $\ell_{1}$-norm or mixed $\ell_{1} / \ell_{2}$-norm based reconstruction algorithms, $\ell_{p}$-norm also brings the dependence on the power in each subband. In this section, we apply a weighted bands constraint to deal with this imbalance. For $q \geq 2 N B$, Equation (22) can be reduced to

$$
\underset{\mathbf{r}_{f}}{\operatorname{minimize}} F_{\sigma}(\xi)=\sum_{i=1}^{N}\left(1-e^{-\left[r_{f}^{*}(i)+v_{i}^{T} \xi\right]^{2} / 2 \sigma^{2}}\right)
$$



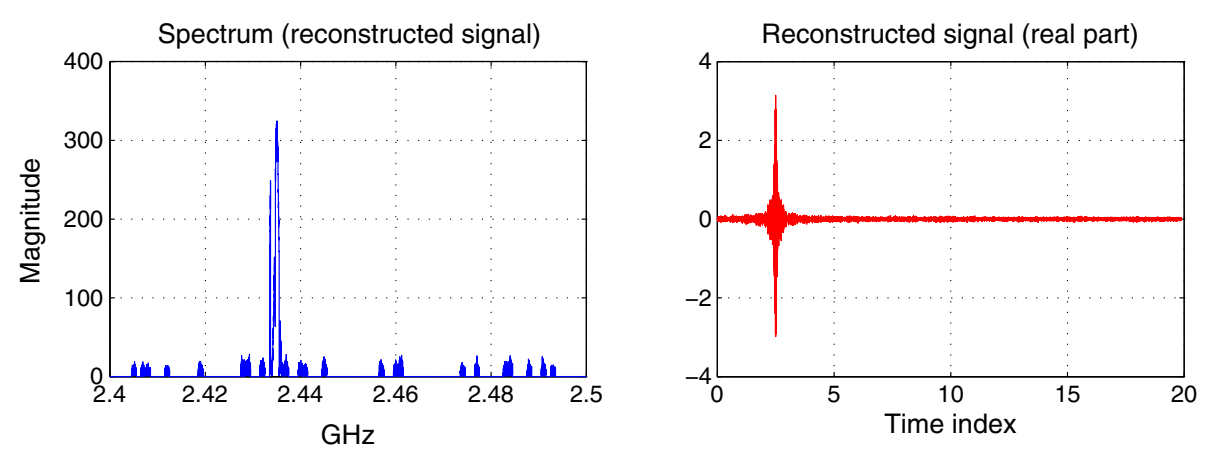

(a) BP (Average squared error: 0.3029)
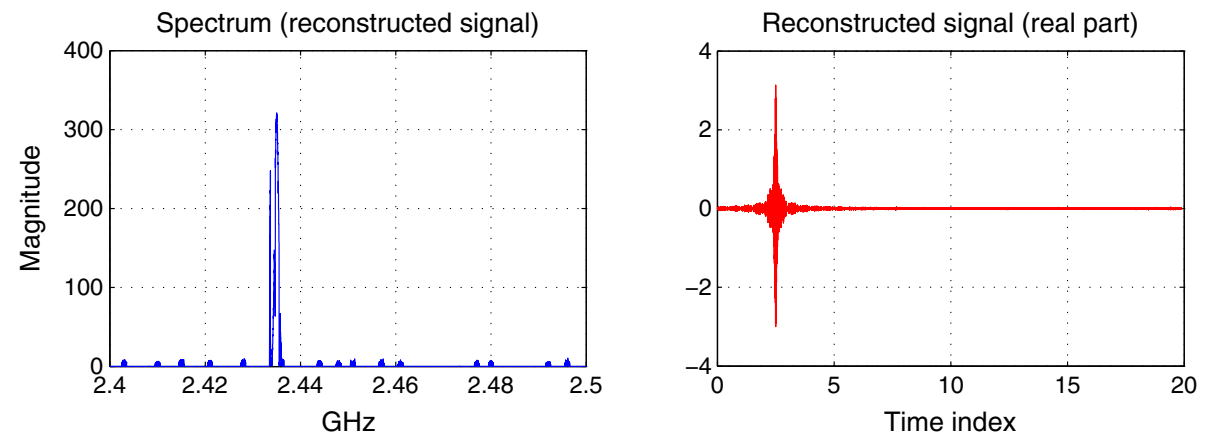

(b) LASSO (Average squared error: 0.0253)


(c) WBSR (Average squarederror: 0.0087)

Figure 3 CS signal reconstruction.

in which $\mathbf{r}_{f}^{*}(i)$ is the $i$ th row of special solution and $\mathbf{v}_{i}^{T}$ denotes the $i$ th row of matrix $\mathbf{V}_{r}$. It can be seen that Equation (25) remains differentiable, so it can be easily solved with gradient descent method.

As analyzed in Section "System models", in wideband spectrum sensing, the blind sensing problem can be formulated as Equation (9), where $r_{1}, r_{2}, \ldots, r_{K}$ are $K$ subsets of $\mathbf{r}$ with different length (block-length), which corresponds to the bands of multi-coset spectrum dividing scheme. It is clear that the power of each band may be different, which depends on the power of the primary user existing in the $i$ th subband. So it can be reformulated as a weighted bands problem:

$$
\underset{\mathbf{r}_{f}}{\operatorname{minimize}} \sum_{i=1}^{K} \sum_{j=1}^{d_{i}} w_{i} \cdot\left(1-e^{-\left[\mathbf{r}_{f}^{*}(j)+v_{j}^{T} \xi\right]^{2} / 2 \sigma^{2}}\right)
$$

The weights $w_{i}, i=1, \ldots, K$ can be calculated according to the existing subband power (ESP) $e_{i}, w_{i}=\frac{1}{e_{i}+\delta}$, here $\delta$ is a small constant (about $10^{-4}$ ) which is used to guarantee $w_{i}$ to be non-convex. The initial condition of the recursive 
relation is $w_{i}=1, i \in\{1, \ldots, K\}$, which means that in the first step all the blocks are weighted equally.

With the increase of the iteration times, larger values of ESP in the $i$ th subband are penalized lighter than smaller values of ESP. A threshold can be applied to terminate the iteration at the proper time, we have

$$
\left\|\mathbf{r}_{f}^{l}-\mathbf{r}_{f}^{l-1}\right\|_{2} \leq \epsilon
$$

in which $\mathbf{r}_{f}^{l}$ is the estimated frequency spectrum at the $l$ th iteration and $\epsilon$ bounds the iteration residual.

By this way, a compressible signal $\mathbf{r}_{f}$ can effectively be reconstructed with measurement $\mathbf{A r}_{f}=\mathbf{y}$. The most innovation feature of WBSR is that it is able to perfectly reconstruct signals without prior information of the sparsity. Compared with previous methods, the advantages of WBSR also are fast, stable, and uniform guarantees.

\section{Simulation}

To evaluate the proposed method, we simulate the system on test signals contaminated by white Gaussian noise. We consider bands in the ISM bands with a frequency range from $2.4-2.4835 \mathrm{GHz}$. In general, $2.4-2.4835 \mathrm{GHz}$ spectrum bands are shared by many wireless devices, such as home microwave oven, wireless sensor networks (Zigbee), WLANs (IEEE 802.11), Bluetooth devices (IEEE 802.15.1), cordless phones, wireless USB device, and so on. In CRNs, the primary users should be tolerant of ISM emission in these bands. The unlicensed second users are able to utilize these bands without causing interference to primary users.

In simulations, we consider a Bluetooth signal in ISM bands, and ignore the baseband protocol for Bluetooth chip. First, we evaluate the performance on 2,048 noisy Bluetooth signals, in which the noise is a white Gaussian noise process. The signal is constructed using the formula (QPSK):

$$
r(t)=\sqrt{E B} \operatorname{sinc}(B(t-\tau)) \cos (2 \pi f(t-\tau))
$$

For simplicity, the energy coefficients $E=1$, the time offsets $\tau=0.5 \mu \mathrm{s}, Q=1$, and $B=2 \mathrm{MHz}$. Because Bluetooth defines 79 channels, each channel being separated by $1 \mathrm{MHz}$ Bluetooth's transmitted signals are spread across this $2.4 \mathrm{GHz}$ band and the specification allows for 1600 frequency hops per second. In Bluetooth-based communications, because the information is spread across a number of frequency channels.

In simulation, we use $\epsilon$ as $10^{-4}$. According to the basic idea of CS theory, the sampling is significantly reduced by $60 \%$. To compare the results, we use BP and LASSO schemes under the same scenario. In Figure 3 the comparison results are given. In each subfigure, the original signal and its spectrum are compared with the reconstructed signal and spectrum. The average squared errors are 0.3029 , 0.0254 , and 0.0087 for BP, LASSO, and WBSR, respectively, which are obtained according to Equation (29)

$$
E_{\mathrm{avr}}=\frac{1}{N} \sum_{i=1}^{N}\left(r_{i}-\hat{r}_{i}\right)^{2}
$$

The performance of spectrum sensing of the proposed scheme is depicted in Figure 4, where the estimated transmit power is shown. It is seen that the PUs start the transmission around $t=400$ with $1 \mathrm{~W}$ of power. Note that the WBSR algorithm can correctly identify the presence of the primary user activities with the LASSO based scheme, which sets noise at $10 \mathrm{~dB}$. It can be seen that the WBSR outperforms BP and LASSO based schemes in spectrum sensing and signal reconstruction.

Compared with the existing schemes as shown in Figure 4, WBSR significantly improves the spectrum sensing speed by ignoring the edge detection phase. The proposed scheme only uses the energy detection to evaluate the weights for each iteration. Therefore, the proposed WBSR outperforms the existing BP and LASSO-based scheme with a much smaller sensing sensitivity and higher accurate spectrum reconstruction for signals, so it might be a promising technique for wideband spectrum sensing in CRN.

In this article, the computational complexity of the proposed algorithm can be measured in terms of the average CPU time that is obtained from a total of 40 trials for typical signals. Two kinds of signals with different lengths are used in simulations: one with 65535, another with 19600. All simulations are performed on a laptop with an Intel T5750 $2 \mathrm{GHz}$ processor and the CPU time was measured by the Matlab (version 2009b) commands tic and toc. Table 2 depicts the simulation results, from which it can be seen that the compressed ratio $(M / N)$ is about $40 \%$. It is noted that the proposed WBSR is more efficient than other algorithms.

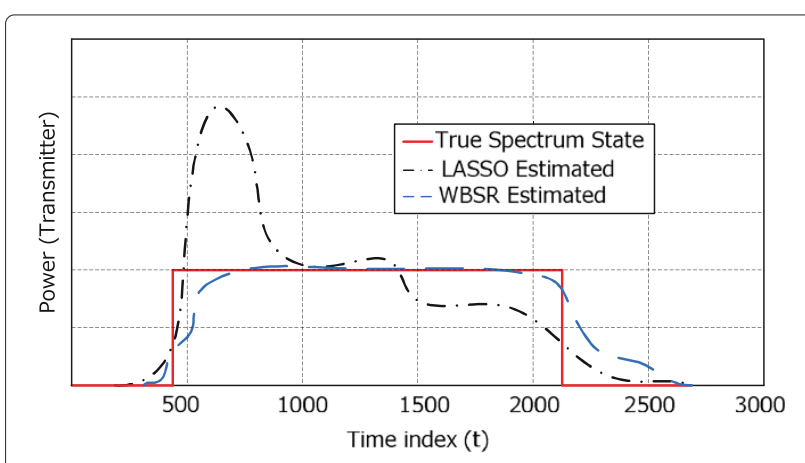

Figure 4 Estimated emitted power levels. 
Table 2 Average CPU time over 40 runs for different algorithm

\begin{tabular}{lccc}
\hline Algorithm & Signal length & $\boldsymbol{M} / \boldsymbol{N}(\%)$ & Average CPU time(s) \\
\hline BP & 65535 & 39.85 & 16.732 \\
LASSO & 65535 & 39.85 & 0.5361 \\
WBSR & 65535 & 39.85 & 0.2132 \\
BP & 19600 & 40 & 6.2863 \\
LASSO & 19600 & 40 & 0.1260 \\
WBSR & 19600 & 40 & 0.0806 \\
\hline
\end{tabular}

In simulations, we compared the proposed $\ell_{p}$-norm minimization scheme with the most popular used $\ell_{1}$ (BP) and $\ell_{2}$ (LASSO) optimizations and the results can be found in Table 2. It is clear that the proposed WBSR is converged much faster than BP and LASSO. Actually, in the existing works, block-sparse spectrum sensing is based on $\ell_{1}$-norm optimization, spectrum-blind reconstruction and LASSO-CWSS are based on $\ell_{2}$-norm optimization, respectively. In this simulation, when we use $\ell_{1}$-norm based optimization for a signal with 65,535 elements, the average CPU time used for reconstruction with $95 \%$ accuracy (or higher) is about $16.732 \mathrm{~s}$. When $\ell_{2}$-norm is used, the average CPU time is $0.5361 \mathrm{~s}$. However for WBSR, the average CPU time is only $0.2132 \mathrm{~s}$ that is much faster than the other two. Similar results can be obtained for signals with different length. In order to further demonstrate the performance of WBSR, we are working to implement the algorithm in practical platform and the results will be reported in the future.

\section{Conclusion}

In this article, we presented an approach that is able to reconstruct the blind bands signals for CRNs without knowing the bands location information, which can improve the spectrum sensing efficiency and reduce the sensing time. In addition, in order to further improve the performance of existing CS-based signal reconstruction algorithm and decrease the complexity, we proposed a weighted $\ell_{p}$-norm $(1<p<1)$ minimization problem to approximate the $\ell_{0}$-norm minimization problem, instead of $\ell_{1}$-norm or $\ell_{1} / \ell_{2}$ mixed minimization in existing signal reconstruction schemes. Simulation results show that the proposed WBSR has a higher spectrum sensing sensitivity and accuracy, and improved reconstruction speed.

\section{Competing interests}

The authors declare that they have no competing interests.

\section{Acknowledgements}

This study was partially supported by the Nation Science Foundation of China (NSFC) under grant numbers 81101118 and 60972038.

\section{Author details}

${ }^{1}$ Key Laboratory of Biomedical Information Engineering of Ministry of Education, Xi'an Jiaotong University, Xi'an 710049, China. ${ }^{2}$ College of
Engineering, Swansea University, Swansea, SA2 8PP, UK. ${ }^{3}$ National Lab of Radar Signal Processing, Xidian University, Xi'an 710072, China.

Received: 2 October 2011 Accepted: 23 July 2012

Published: 3 October 2012

\section{References}

1. M Mishali, YC Eldar, Wideband spectrum sensing at sub-Nyquist rates. http://arxiv.org/abs/1009.1305

2. FCC, Facilitating opportunities for flexible, efficient and reliable spectrum use employing cognitive radio technologies. FCC Report and Order FCC-05-57A1, 266-267 (2005)

3. Z Tian, GB Giannakis, Compressed sensing for wideband cognitive radios. in IEEE International Conference on Acoustics, Speech and Signal Processing, vol. 4, (Honolulu, Hawaii, USA, 2007), pp. 1357-1360

4. T Agrawal, V Lakkundi, A Griffin, P Tsakalides, Compressed sensing for OFDM UWB systems. in IEEE Radio and Wireless Symposium, vol. 1 (Phoenix, AZ, USA, 2011), pp. 190-193

5. M Mishali, YC Eldar, From theory to practice: sub-Nyquist sampling of sparse wideband analog signals. IEEE J. Sel. Topics Signal Process. 4, 375-391 (2010)

6. M Mishali, YC Eldar, Blind multiband signal reconstruction: compressed sensing for analog signals. IEEE Trans. Signal Process. 57, 993-1009 (2009)

7. Z Khalaf, A Nafkha, J Palicot, Blind spectrum detector for cognitive radio using compressed sensing. in IEEE Global Telecommunications Conference (Globecom 2011) vol. 1. (San Diego, CA, USA, IEEE, 2011), pp. 1-5

8. Y Wang, A Pandharipande, YL Polo, G Leus, Distributed compressive wide-band spectrum sensing. in Information Theory and Applications Workshop vol. 1. Huston, Texas, USA, IEEE, 2009), pp. 178-183

9. Y Liu, Q Wan, Generalized block sparse constraint based compressive wideband spectrum spectrum sensing for cognitive radio. http://arxiv. org/abs/1006.2055

10. G Rath, C Guillemot, Sparse approximation with an orthogonal complementary matiching pursuit algorithm. in IEEE International Conference on Acoustics, Speech and Signal Processing vol.1. (Huston, Texas, USA, IEEE, 2009), pp. 178-183

11. J Chen, X Huo, Collaborative compressed spectrum sensing: what if spectrum is not sparse. Electron Lett. 47, 519-520 (2011)

12. Z Tian, G Leus, V Lottici, Joint dynamic resource allocation and waveform adaptation for cognitive networks. IEEE J. Sel. Areas Commun. 29, 443-454 (2011)

13. L Gueguen, B Sayrac, Sensing in cognitive radio channels: a theoretical perspective. IEEE Trans. Wirel. Commun. 8, 1194-1198 (2009)

14. W Zhang, K Letaief, Cooperative spectrum sensing with transmit and relay diversity in cognitive radio networks. IEEE Trans. Wirel. Commun. 7, 4761-4766 (2008)

15. SS Chen, Basis pursuit. PhD thesis: Department of Statistics, Stanford University, 1995)

16. SChen, D Donoho, M Saunders, Atomic decomposition by basis pursuit. SIAM J. Sci. Comput. 20, 31-61 (1999)

17. E Candes, TTao, The Dantzig selector: statistical estimation when $p$ is much larger than $\boldsymbol{n}$. Ann. Stat. 34, 2313-2351 (2007)

18. SG Mallat, Z Zhang, Matching pursuit in a time-frequency dictionary. IEEE Trans. Signal Process. 41, 3397-3415 (1993)

19. J Tropp, AC Gilbert, signal recovery from random measurements via orthogonal matching pursuit. IEEE Trans. Inf. Theory. 53, 4655-4666 (2007)

20. D Needell, R Vershynin, Uniformuncertainty principle signal recovery via regularized orthogonal matching pursuit. Found Comput. Math. $\mathbf{9}$, 317-334 (2009)

21. D Needell, JA Tropp, CoSaMP: iterative signal recovery from incomplete and inaccurate samples. Harmonic Anal. Appl. Comput. 26, 301-321 (2008)

22. R Tibshirani, Regression shrinkage and selection via the LASSO. J. R. Stat. Soc. B. 58, 267-288 (1996)

23. T Zhang, Sparse recovery with orthogonal matching pursuit under RIP. IEEE Trans. Inf. Theory. 57, 6215-6221 (2011)

24. H Mohimani, M Babie-Zadeh, C Jutten, A fast approach for overcomplete sparse decomposition based on smoothed $l_{0}$-norm. IEEE Trans. Signal Process. 57, 289-301 (2009) 
25. S Li, X Wang, J Wang, Manifold learning-based automatic signal identification in cognitive radio networks. IET Commun. 6, 955-963 (2012)

26. Z Tian, Y Tafesse, BM Sadler, Cyclic feature detectionwith sub-Nyquist sampling for wideband spectrum sensing. IEEE J. Sel. Topics Signal Process. 6, 58-70 (2012)

27. $S \mathrm{Li}, \mathrm{X}$ Wang, Polychromatic set theory-based spectrum access in cognitive radios. IET Commun. 6, 909-916 (2012)

28. A Antoniou, WS Lu, Practical Optimization: Algorithms and Engineering Applications: (Springer, Heidelberg, 2006)

29. JK Pant, WS Lu, A Antoniou, Reconstruction of sparse signals by minimizing a re-weighted approximate $l_{0}$-norm in the null space of the measurement matrix. in 53rd IEEE International Midwest Symposium on Circuits and Systems (MWSCAS). (vol. 1 Taipei, IEEE 2010), pp. 3325-3328

doi:10.1186/1687-1499-2012-306

Cite this article as: Li et al.: Efficient blind spectrum sensing for cognitive radio networks based on compressed sensing. EURASIP Journal on Wireless Communications and Networking 2012 2012:306.

\section{Submit your manuscript to a SpringerOpen ${ }^{\circ}$ journal and benefit from:}

- Convenient online submission

- Rigorous peer review

- Immediate publication on acceptance

- Open access: articles freely available online

- High visibility within the field

- Retaining the copyright to your article

Submit your next manuscript at $\gg$ springeropen.com 